\title{
Olanzapine-induced early cardiovascular effects are mediated by the biological clock and prevented by melatonin
}

\author{
Francisco Romo-Nava ${ }^{1,2,3}$ (D) | Frederik N. Buijs ${ }^{1,4}$ | Marcela Valdés-Tovar ${ }^{5}$ | \\ Gloria Benítez-King $^{5}$ | MariCarmen Basualdo ${ }^{1}$ | Mercedes Perusquía $^{6}$ | \\ Gerhard Heinze $^{2}$ | Carolina Escobar ${ }^{7}$ | Ruud M. Buijs ${ }^{1}$
}

${ }^{1}$ Hypothalamic Integration Mechanisms Laboratory, Departamento de Biología Celular y Fisiología, Instituto de Investigaciones Biomédicas, Universidad Nacional Autónoma de México (UNAM), México city, DF, México

${ }^{2}$ Departamento de Psiquiatría y Salud Mental, Facultad de Medicina, UNAM, México city, DF, México

${ }^{3}$ Division of Bipolar Disorder Research, Department of Psychiatry and Behavioral Neuroscience, College of Medicine, University of Cincinnati, Cincinnati, OH, USA

${ }^{4}$ Netherlands Institute for Neuroscience, an Institute of the Royal Netherlands Academy of Arts and Sciences, Amsterdam, BA, The Netherlands

${ }^{5}$ Laboratorio de Neurofarmacología, Subdirección de Investigaciones Clínicas, Instituto Nacional de Psiquiatría Ramón de la Fuente Muñiz, México city, DF, México

${ }^{6}$ Endocrinology of Reproduction

Laboratory, Departamento de Biología

Celular y Fisiología, Instituto de

Investigaciones Biomédicas UNAM,

México city, DF, México

${ }^{7}$ Departamento de Anatomía, Facultad de Medicina, UNAM, México city, DF, México

\section{Correspondence}

Ruud M. Buijs, Hypothalamic Integration Mechanisms Laboratory, Departamento de Biología Celular y Fisiología, Instituto de Investigaciones Biomédicas, UNAM. Ciudad Universitaria, México City, México. Email: ruudbuijs@gmail.com

Funding information

Support was provided by DGAPA IG-

200417; CONACYT 220598. R.M.B., and

SEP-CONACYT No. 178075 (G.B.K.).

\begin{abstract}
Second generation antipsychotics (SGA) are associated with adverse cardiometabolic side effects contributing to premature mortality in patients. While mechanisms mediating these cardiometabolic side effects remain poorly understood, three independent studies recently demonstrated that melatonin was protective against cardiometabolic risk in SGA-treated patients. As one of the main target areas of circulating melatonin in the brain is the suprachiasmatic nucleus ( $\mathrm{SCN})$, we hypothesized that the SCN is involved in SGA-induced early cardiovascular effects in Wistar rats. We evaluated the acute effects of olanzapine and melatonin in the biological clock, paraventricular nucleus and autonomic nervous system using immunohistochemistry, invasive cardiovascular measurements, and Western blot. Olanzapine induced c-Fos immunoreactivity in the SCN followed by the paraventricular nucleus and dorsal motor nucleus of the vagus indicating a potent induction of parasympathetic tone. The involvement of a SCN-parasympathetic neuronal pathway after olanzapine administration was further documented using cholera toxin-B retrograde tracing and vasoactive intestinal peptide immunohistochemistry. Olanzapine-induced decrease in blood pressure and heart rate confirmed this. Melatonin abolished olanzapineinduced SCN c-Fos immunoreactivity, including the parasympathetic pathway and cardiovascular effects while brain areas associated with olanzapine beneficial effects including the striatum, ventral tegmental area, and nucleus accumbens remained activated. In the SCN, olanzapine phosphorylated the GSK-3 $\beta$, a regulator of clock activity, which melatonin prevented. Bilateral lesions of the SCN prevented the effects of olanzapine on parasympathetic activity. Collectively, results demonstrate the SCN as a key region mediating the early effects of olanzapine on cardiovascular function and show melatonin has opposing and potentially protective effects warranting additional investigation.
\end{abstract}

\section{K E Y W O R D S}

blood pressure, cardiovascular, melatonin, olanzapine, suprachiasmatic nucleus 


\section{1 | INTRODUCTION}

Second generation antipsychotics (SGA) induce severe adverse cardiometabolic effects that affect millions of patients and families by decreasing drug adherence or dramatically increasing their health burden with metabolic comorbidity $^{1}$. Olanzapine is a SGA effectively used to treat patients with mental disorders, but unfortunately induces adverse cardiometabolic effects including weight gain, fat mass, glucose, insulin, and lipid increases ${ }^{2-4}$. Interestingly, in rats and humans, acute SGA treatment may induce hypotension ${ }^{5-7}$, while long-term SGA treatment is associated with increased blood pressure ${ }^{8}$. In consequence, they have become an enormous health problem in a population in which the risk of metabolic abnormalities is two to threetimes greater than that of the general population ${ }^{4}$. These patients also suffer a premature death 11 to 20 years earlier, largely due to cardiovascular disease ${ }^{9,10}$. The SGA-induced cardiometabolic effects are also present in acute administration studies in healthy individuals, indicating that metabolism changes are independent of caloric intake and disease related factors ${ }^{3,11}$. Even though intense research efforts during the past decade have focused on providing a plausible mechanism for their genesis and prevention, an integral explanation is lacking and the therapeutic options to prevent them are limited and urgently needed.

Metabolism is influenced through central hypothalamic mechanisms that involve the biological clock located in the suprachiasmatic nucleus ( $\mathrm{SCN}$ ) and the autonomic nervous system (ANS) ${ }^{12-14}$. Disturbances of these mechanisms have been associated with metabolic problems such as the metabolic syndrome ${ }^{14,15}$, which resembles SGA-induced cardiometabolic effects.

In a recent study, the pineal hormone melatonin, which signals darkness to the SCN depressing its activity ${ }^{16}$, attenuated olanzapine-induced weight gain in rats ${ }^{17}$. Hereafter in randomized controlled trial (RCT), we demonstrated that melatonin attenuates SGA-induced cardiometabolic effects in patients, particularly those with bipolar disorder without affecting the psychopathological outcome ${ }^{18}$. In two other RCT, melatonin mitigated olanzapine-induced cardiometabolic effects in patients diagnosed with schizophrenia ${ }^{19}$ and bipolar disorder ${ }^{20}$.

The SCN is strongly involved in metabolic and cardiovascular control ${ }^{12}$. As one of the main targets of circulating melatonin in the brain is the $\mathrm{SCN}^{16,21}$, these basic and clinical results could suggest the involvement of central mechanisms in SGA-induced cardiometabolic effects. Therefore, we examined in a rat model the early effects of olanzapine on hypothalamic nuclei relevant for metabolic regulation and uncovered a hitherto unknown action of olanzapine on the biological clock resulting in a decrease in blood pressure; which was prevented by melatonin. These findings identify a novel $\mathrm{SCN}$-centered mechanism for olanzapine cardiometabolic adverse effects and their prevention by melatonin without tampering the therapeutic effects.

\section{2 | METHODS AND MATERIALS}

\section{1 $\quad$ Ethical statement for animal experimentation}

All experiments were carried out with the approval of the research ethics committee of the Institute of Biomedical Research at the Universidad Nacional Autonóma de México (UNAM) and were conducted in strict accordance with current legislation and technical specifications for production, care and use of laboratory animals (Norma Oficial Mexicana NOM- 062 -ZOO- 1999).

\subsection{Immunohistochemistry for c-Fos}

Male Wistar rats (200-250 g) were used to evaluate the effect in the brain and autonomic nervous system of acute administration of olanzapine (Zyprexa ${ }^{\circledR}$ powder for solution, Lilly USA, LLC, Indianapolis, USA) and melatonin (Sigma-Aldrich product No. M5250, Saint Louis, MO 63103, USA). Animals were in light-dark cycles of 12 hour: 12 hour (Lights on at 07:00 hour, lights off at 19:00 hour) with ad libitum food and water. Rats received a single subcutaneous dose of olanzapine $(2.5 \mathrm{mg} / \mathrm{kg})$, olanzapine $(2.5 \mathrm{mg} / \mathrm{kg})+$ melatonin $(2.5 \mathrm{mg})$, melatonin $(2.5 \mathrm{mg}$ ), or saline $(\mathrm{NaCl} 0.9 \%)$ at ZT11 (one hour before dark onset) and were sacrificed at ZT14. Olanzapine and melatonin doses fall within the range used for previously published studies in rats ${ }^{22-24}$. Olanzapine and melatonin were dissolved in a $20 \%$ ethanol and saline solution (final volume of administration: $0.5 \mathrm{~mL}$ ). Saline and olanzapine only groups received the same amount of ethanol. (Fig. S1).

Animals were sacrificed 3 hours after drug administration (ZT14). To obtain the brains, animals were anesthetized with an overdose of intraperitoneal (ip) pentobarbital $(210 \mathrm{mg} / \mathrm{kg})$ and perfused with an intracardiac infusion of $250 \mathrm{ml}$ saline solution $(0.9 \% \mathrm{NaCl})$, followed by $200 \mathrm{ml} 4 \%$ paraformaldehyde prepared in $0.1 \mathrm{M}$ phosphate buffer, $\mathrm{pH}$ 7.2. Brains and spinal cords were removed and placed in $4 \%$ paraformaldehyde for 24 hours and subsequently cryo-preserved in $30 \%$ sucrose for 72 hour. The brains were frozen and cut in $40 \mu \mathrm{m}$ coronal plane slices and maintained in culture dishes with a $0.1 \mathrm{M}$ phosphate buffer, $\mathrm{pH}$ 7.2. The primary antibody for c-Fos (1:40,000; Calbiochem, \#PC38, San Diego, CA, USA) used as a marker of neural activity and was incubated for 1 hour. at room temperature and 48 hours at $4^{\circ} \mathrm{C}$. After extensive washing, donkey anti-rabbit biotinylated secondary antibody (1:200, Jackson Immunoresearch, West Grove, PA, USA) was applied and incubated for 2 hour. The avidin-peroxidase complex (ABC 9:1000; Vectastain, Vector Laboratories, Burlingame, CA, USA) was applied, and 
finally, the reaction was visualized with diaminobenzidine in PBS $(50 \mathrm{mg} / 100 \mathrm{~mL}, \mathrm{pH} 7.2)$ combined with $0.003 \%$ peroxide and $0.01 \%$ nickel. The sections were mounted on gelatinized slides and cover slipped with Entellan ${ }^{\circledR}$ (EMD Millipore Corp., Billerica, MA, USA). Coronal sections of each side, in each nucleus or region studied, were selected using the following distance from bregma coordinates: $\mathrm{SCN}(-0.60 \mathrm{~mm})$; paraventricular nucleus (PVN; $-1.72 \mathrm{~mm})$; dorsal motor nucleus of the vagus (DMV; $-14.40 \mathrm{~mm}$ ); intermediolateral column (IML; T3); striatum $(0.96 \mathrm{~mm})$; ventral tegmental area $(-5.04 \mathrm{~mm})$; and nucleus accumbens $(0.96 \mathrm{~mm}){ }^{25}$.

\subsection{Neuronal tracer injection}

To confirm that olanzapine's effects involve the activation of a SCN-PVN-DMV neuronal pathway, the retrograde tracer cholera toxin-B (CtB) was injected into the DMV to analyze co-localization with c-Fos in the PVN pre-autonomic neurons after olanzapine administration and the presence of VIP projections from the $\mathrm{SCN}$ to them.

After anesthesia, the rats were placed in a David Kopf stereotaxic frame with the head fixed at $45^{\circ}$. Dissection of the dura and arachnoid to expose the dorsal surface of the bone at the level of the area postrema was performed. The head of the rat was placed so that the micropipette was aligned perpendicularly to the medulla oblongata. CtB $(50 \mu \mathrm{l}, 0.5 \%)$ was injected, by means of a glass micropipette with a $0.02 \mu \mathrm{l}$ tip, unilaterally into the dorsal vagal complex by pressure $(10 \mathrm{mbar}$, for 5 seconds). Following 10 days of recovery, animals received the acute subcutaneous administration of olanzapine and were sacrificed following the aforestated procedure. Injection accuracy was confirmed by $\mathrm{CtB}$ immunostaining. (Fig. S2).

Immunohistochemistry in PVN coronal sections was performed with c-Fos as described above. Immunohistochemistry for vasoactive intestinal peptide (VIP) to observe SCN projections to the PVN pre-autonomic neurons was then performed sequentially incubating the primary antibody for (rabbitVIP) in a 1:2000 dilution for 1 hour at room temperature and 24 hours at $4^{\circ} \mathrm{C}$. Secondary antibody, AB, and visualization with $\mathrm{DAB}$ procedures were followed as previously described. On the same PVN sections, CtB immunohistochemistry was performed using polyclonal rabbit anti-CtB immunoglobulin (1:1000, Sigma-Aldrich; No. C3062) to visualize PVN neurons retrogradely filled from DMV CtB injections. After incubating $24 \mathrm{hrs}$ at $4{ }^{\circ} \mathrm{C}$, secondary antibody, $\mathrm{AB}$, and visualization with $\mathrm{DAB}$ procedures were followed as described above without nickel to obtain a brown-reddish stain.

\section{4 | Hemodynamic Measurements}

Blood pressure and heart rate were measured to evaluate the functional relevance of the effect of olanzapine and melatonin on the parasympathetic nervous system. To evaluate the involvement of the SCN over the cardiovascular effects of olanzapine, we included a group of bilaterally SCN lesioned $(\mathrm{SCNxx})$ animals and measured the immediate effects of olanzapine on blood pressure and heart rate.

Blood pressure and heart rate measurements were made through a femoral artery catheter. Cannulation of the femoral artery was performed as described elsewhere ${ }^{26}$. In brief, rats were anesthetized with ip. urethane $(1.5 \mathrm{~g} / \mathrm{kg})$ diluted in $2 \mathrm{~mL}$ of saline solution $(0.9 \% \mathrm{NaCl})$. Rats were placed in supine position, and fur around the inguinal surgical region was shaved. A small $(1-2 \mathrm{~cm})$ incision along the natural angle of the leg was placed and femoral vein and artery exposed through blunt dissection. The femoral artery was separated from the vein, nerve, and surrounding tissue and retractors placed to fully view the artery and vein. Folded sterile 4.0 silk was placed under the femoral artery and cut to obtain proximal and distal silk pieces. The distal silk piece was pulled caudally and the proximal piece cranially to allow hemostatic control of the artery. A small incision was made on the artery section between the silk pieces; fine tip forceps were inserted into the incision and used to allow the insertion of the catheter. The catheter was pushed into the artery and proximally fixed. The functionality of the catheter was checked and two hours after surgery, connected to a blood pressure transducer.

\section{5 | Bilateral lesion of the suprachiasmatic nucleus}

The SCN lesion technique has been previously described ${ }^{27}$. In short, animals were anesthetized with IP. ketamine/xylazine (90 mg and $10 \mathrm{mg} / \mathrm{kg}$ ) and placed on a stereotaxic surgery frame (Model 900, David Kopf). Coordinates used to lesion the SCN were $2.2 \mathrm{~mm}$ posterior to bregma, $0.9 \mathrm{~mm}$ ventral, and $0.2 \mathrm{~mm}$ lateral. Lesions were performed using epoxy-insulated insect pins $(0.20 \mathrm{~mm})$ with excoriated tip. Direct electrical current for 30 seconds was applied bilaterally. Rats recovered from surgery for 2 weeks with successful lesions confirmed by actigraphic registration and SCN VIP immunostaining as described above. (Fig. S3).

\subsection{Hemodynamic registration}

Blood pressure and heart rate measurements were recorded from the artery catheter by a pressure transducer (P23 XL, Grass Instrument, Quincy, MA, USA) connected to a MP150 Research System, and the data were analyzed using AcqKnowledge software (Biopac Systems Inc., Goleta, CA, USA). For the purpose of this experiment, baseline tracings, as well as saline, olanzapine and melatonin were sequentially administered ip. at the previously described doses to ensure quantifiable responses of hemodynamic parameters. Data were obtained from three experimental groups of animals, with minutes indicating the time data were recorded after 
drug administration (IP.). Group 1 (olanzapine): Baseline values were obtained (10 minute) followed by $0.5 \mathrm{ml}$ saline (10 minute) and a single olanzapine $(0.5 \mathrm{mg} / \mathrm{kg})$ injection (60 minute). Group 2 (melatonin + olanzapine): Ensuing baseline recording (10 minute), a $0.5-\mathrm{ml}$ saline injection (10 minute) was followed by a single melatonin $(2.5 \mathrm{mg})$ IP. injection (10 minute) and successively olanzapine ip. was given (60 minute). Group 3 (SCNxx animals + olanzapine): Baseline tracing (10 minute) preceded $0.5 \mathrm{ml}$ saline I.P. (10 minute), followed by a single olanzapine I.P. injection (60 minute).

\subsection{Western blot}

For nocturnal (ZT11) drug administration for saline, olanzapine, olanzapine+melatonin, and melatonin, the protocol was followed as described above ( $n=5$ animals/experimental group). Animals were sacrificed at ZT14 by decapitation. The suprachiasmatic nucleus was immediately obtained, preserved in RIPA buffer with protease and phosphatase inhibitors, and frozen at $-72^{\circ} \mathrm{C}$. Tissue was homogenized, and total protein concentration was determined by Lowry's assay $^{28}$. Equal amounts of protein were loaded into polyacrylamide gels and phosphorylated, as well as total GSK-3 $\beta$ was evaluated by Western blot. Glyceraldehyde-3-phosphate dehydrogenase GAPDH was also immunodetected as load control. In short, proteins were separated by one-dimensional SDS-PAGE in $10 \%$ polyacrylamide gels ${ }^{29}$ and transferred according to Towbin's procedure ${ }^{30}$. Phosphorylated GSK-3 $\beta$ (Ser9) was identified with an anti-phospho-GSK-3 $\beta$ antibody (1:1300, Cell Signaling Technology Inc., \#9336, Danvers, MA, USA) and a peroxidase-conjugated donkey anti-rabbit IgG (Jackson Immunoresearch, \#711-005-152) diluted 1:10,000. Detection was performed by chemiluminiscence $^{31}$. After mild stripping, total GSK-3 $\beta$ and GAPDH were identified using antibodies for GSK-3 $\beta$ (1:1400, Santa Cruz Biotech, \#SC-9166, Dallas, Tx, USA) and GAPDH (1:20,000, EMD Millipore Corp., MAB374, Billerica, MA, USA). Respective peroxidase-conjugated secondary antibodies were donkey anti-rabbit $\operatorname{IgG}(1: 20,000$, Jackson Immunoresearch, \#711-005-152) and goat anti-mouse IgG (1:100,000, Jackson Immunoresearch, \# 205-005-108). Samples from five independent experiments were obtained, and Western blots were assayed by triplicate.

Fluorogram images were obtained with a densitometer (GS-800, Bio-Rad, Hercules, CA, USA), and semiquantitative amounts of phosphorylated GSK-3B, total GSK-3 $\beta$, and GAPDH were estimated by densitometry with ImageJ software (Version 1.38X, NIH, Bethesda, MD, USA). Each fluorogram was analyzed in 8-bit type.jpg picture formats. The plot lane tool in ImageJ was used to obtain integrated optical density (OD) values from immunoreactive bands for each experimental group.

\section{8 $\quad$ Statistical Analysis}

The number of c-Fos immunoreactive nuclei was calculated by semi-automatic quantification of the region of interest with ImageJ software (Version 1.38X, NIH, Bethesda, MD, USA) using at least two microphotographs (jpg format) of coronal sections of each side, in each nucleus or region studied. For between-group comparisons ( $n=4$ per group), we considered the average c-Fos immunoreactivity (IR) count for each region and analysis was conducted using ANOVA with Bonferroni's post hoc test for pair-wise comparisons accordingly.

Blood pressure and heart rate analysis were performed using a data acquisition device with AcqKnowledge software (model MP100, Biopac Systems, Goleta, CA, USA) and saved to a personal computer for offline analysis with the use of a Biopac System ECG 100C preamplifier. Ten-minute tracings of mean blood pressure values for baseline, saline, olanzapine, or melatonin injections were obtained. Changes in mean baseline values for systolic, diastolic, and mean arterial blood pressure were calculated for each tracing segment of ten minutes in each experimental group. Heart rate analysis was performed using maximum values in five 2- minute segments for each ten-minute tracing to clear noise from registrations; with the mean for each ten-minute tracing calculated using these values. Mean changes in heart rate, compared to baseline values, were then calculated for saline, olanzapine and/or melatonin injections. Repeatedmeasures ANOVA Bonferroni's post hoc test was used for pairwise comparisons at each time point (10- minute segments).

For Western blot analysis, densitometric analysis of the bands expressed as the optical density in A.U. of total GSK-3 $\beta$ pGSK- $3 \beta$, GAPDH, and the ratio of pGSK- $3 \beta$ to total pGSK- $3 \beta$ (pGSK-3 $\beta /$ Total pGSK-3 $\beta$ ratio) were used for between-group comparisons using one-way ANOVA. All statistical tests were two-tailed and considered significant at $P$ level $<.05$.

\section{3 | RESULTS}

\section{1 | The suprachiasmatic nucleus is activated by olanzapine}

With previous indirect indications that olanzapine may affect the biological clock $^{32}$ and the glycogen synthase kinase- $3 \beta$ (GSK-3ß), which is a regulator of the clock's activity ${ }^{33}$, we examined the effect of a single subcutaneous dose of olanzapine on brain activation. Olanzapine was administered to male Wistar rats (200-250grs) at the beginning of the active phase (night) and the brain obtained $3 \mathrm{hrs}$ later, whereby cFos detection was used as a marker of neuronal activity. The acute peripheral administration of olanzapine induced in the SCN a fourfold increase in the number of c-Fos IR nuclei as compared to saline. The co-administration of olanzapine with melatonin, a hormone able to synchronize the clock by inhibiting its activity ${ }^{16}$, prevented this effect (Figure 1). 

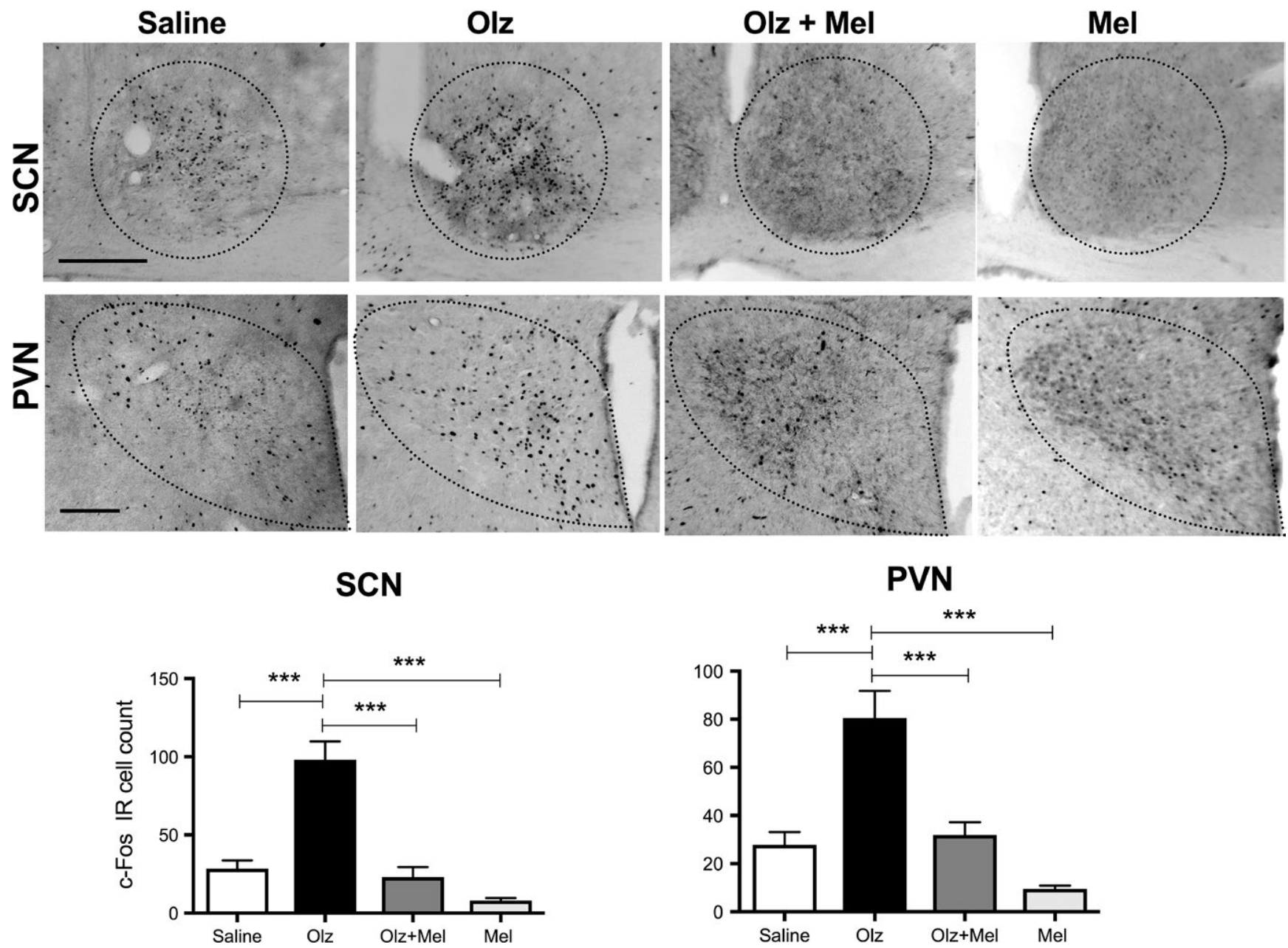

F I G U R E 1 Olanzapine induces neuronal activation in the suprachiasmatic nucleus (SCN) and paraventricular nucleus (PVN) that melatonin prevents. Panel with representative microphotographs of the SCN (top row) and PVN (bottom row) with c -Fos immunoreactivity as a marker for neuronal activity. The left column shows rats treated with saline, followed by a column of olanzapine (Olz), olanzapine + melatonin $(\mathrm{Olz}+\mathrm{Mel})$ and melatonin (Mel) treated rats. Bars represent the mean \pm SEM of c-Fos IR nuclei count in the SCN and the PVN. N=4 animals/group. One-way ANOVA analysis showed a significant between-group differences for SCN $(F=21.27, d f=3, P<.0001)$ and $\mathrm{PVN}(F=16.80, d f=3, P<.0001)$; *** $P<.0001$, Bonferroni's posthoc test for pair-wise comparisons Scale bar is $100 \mu \mathrm{m}$

\section{2 | Olanzapine activates the parasympathetic autonomic axis which is prevented by melatonin}

The acute administration of olanzapine also induced a significantly increased activation in the PVN and DMV, both output pathways from the SCN to the autonomic nervous system (ANS). Similar to the SCN, the co-administration of olanzapine with melatonin prevented this effect (Figure 2). In the spinal cord, olanzapine did not induce an activation of sympathetic motor neurons in the IML (Figure 2). These results indicate that olanzapine selectively increases the activity of the parasympathetic and not the sympathetic branch of the ANS. As the SCN directly signals to the PVN modulating its autonomic output ${ }^{34}$ and melatonin receptors are densely present in the SCN and not in the PVN or $\mathrm{DMV}^{21,35}$, these results suggest that olanzapine activates directly the SCN, which consequentially activates the PVN and DMV.

\section{3 | Melatonin does not impair olanzapine-induced activation in the ventral striatum pathway}

As expected, the administration of olanzapine also induced an activation of the nucleus accumbens, striatum, and ventral tegmental area (VTA), brain areas associated with its therapeutic effect, that do not receive direct input from the $\mathrm{SCN}^{36,37}$. In contrast with our observation in the $\mathrm{SCN}$, the co-administration of melatonin and olanzapine did not alter the activation pattern in the striatum and VTA as observed with olanzapine alone. Moreover, in the nucleus accumbens, the co-administration of melatonin with olanzapine induced a significant increase in cFos activation as compared to the administration of olanzapine alone. These results confirm olanzapine-induced activation of brain regions involved in its therapeutic action and etiology of psychiatric disorders for which it is prescribed ${ }^{36,37}$. Melatonin does not counteract this therapeutic action, and, as seen in the 


\section{Saline}
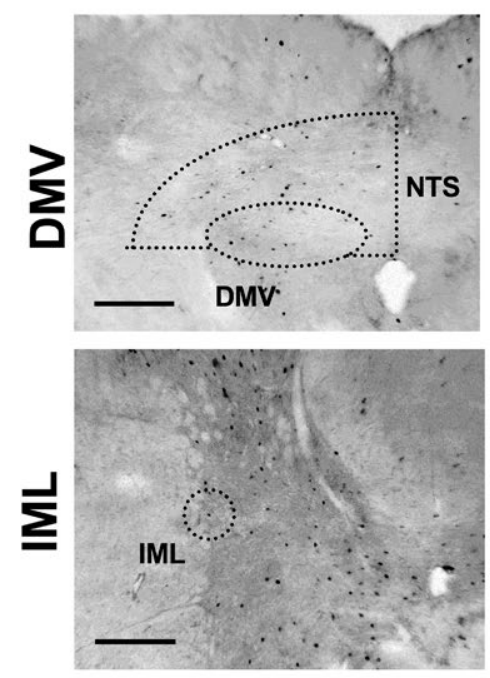

Olz
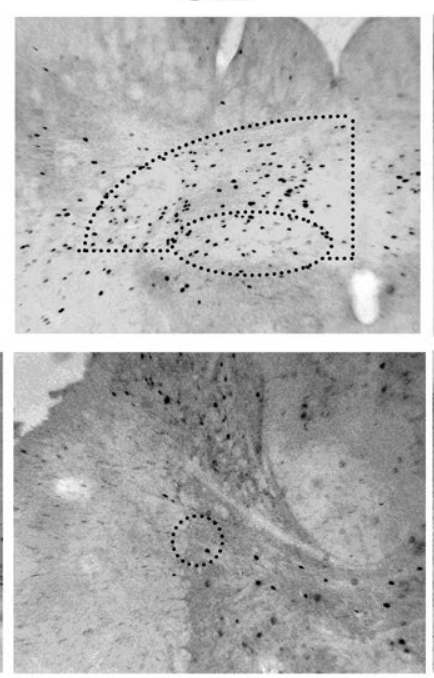

DMV

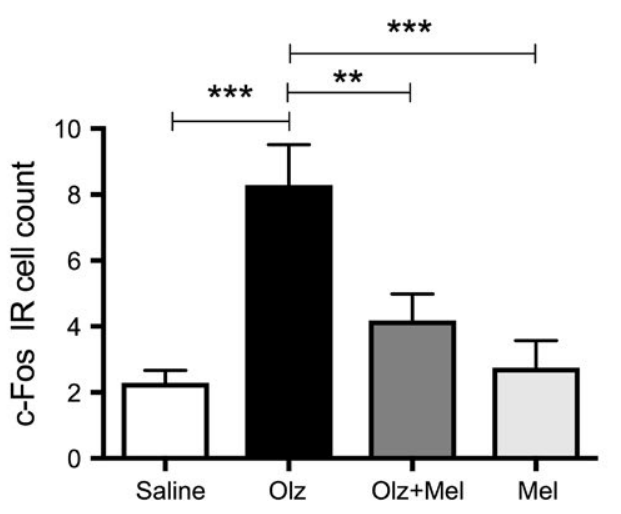

$\mathrm{Olz}+\mathrm{Mel}$
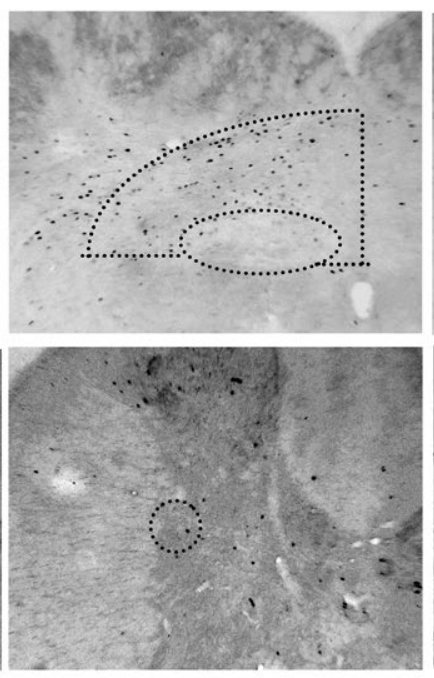

Mel
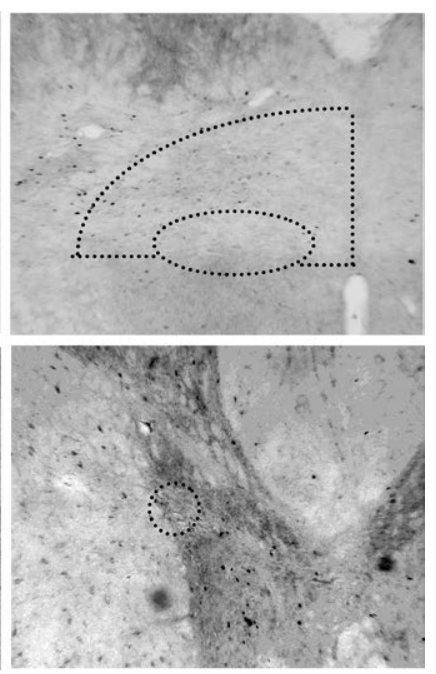

IML

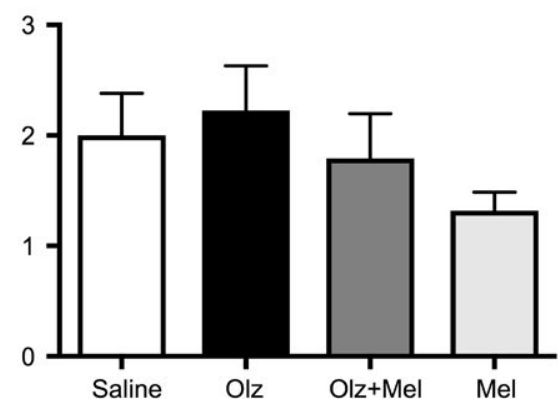

F I G URE 2 Olanzapine induces activation of the dorsal motor nucleus of the vagus (DMV) which is prevented by co-administration of melatonin. Representative microphotographs of the DMV (top) and intermediolateral column (IML; bottom row) with c-Fos immunoreactivity (IR). The left column demonstrates rats treated with saline, followed by a column of olanzapine, olanzapine + melatonin and melatonin only treated rats; $\mathrm{N}=4$ animals/group. Bars represent the mean \pm SEM count of c-Fos IR nuclei in the DMV and the IML. One-way ANOVA analysis showed a significant between-group difference for DMV c-Fos $(F=9.028, d f=3, P<.0001)$. $* * P<.001$ and $* * * P<.0001$; Bonferroni's post- hoc test for pair-wise comparisons. Nucleus of the tractus solitarius (NTS). Scale bar is $100 \mu \mathrm{m}$

nucleus accumbens, it may even potentiate its action (Figure 3). This concurs with clinical observations that co-administration of melatonin with an SGA does not impair its beneficial effects and may even enhance them ${ }^{18-20}$.

Furthermore, olanzapine induced an activation of the nucleus of the tractus solitarius (NTS), which also receives input from sensory pathways indicating that olanzapine also exerts effects on peripheral organs. Melatonin did not prevent this activation (Figure 2 and Fig. S4), supporting a central, rather than peripheral effect of melatonin to prevent the action of olanzapine over the SCN, PVN, and DMV.

\section{4 | Olanzapine activates the parasympathetic pathway via the suprachiasmatic nucleus}

The pattern of c-Fos activation in the PVN suggested that mainly autonomic neurons might be activated. In view of the activation of the NTS and DMV, we decided to place a retrograde tracer into the dorsal vagal complex and confirm activation of the retrogradely labeled neurons. CtB labeled neurons in the PVN showed a high coincidence with c-Fos when olanzapine was given and this response was higher in the autonomic and parvocellular PVN as compared to the magnocellular nuclei (Fig. S2 and Table $\mathrm{S} 1$ ). Some of these activated CtB labeled neurons showed input from SCN neurons as visualized by vasoactive intestinal peptide (VIP) immunohistochemical staining confirming a SCN-PVN-DMV neural pathway, activated by olanzapine.

Parasympathetic activity plays a key role in blood pressure regulation, adiposity and metabolic activity ${ }^{38}$. Acutely, olanzapine induces a potent increase of parasympathetic tone translated into a decrease in blood pressure and heart rate in rats ${ }^{7}$ and postural hypotension in humans ${ }^{5}$. 


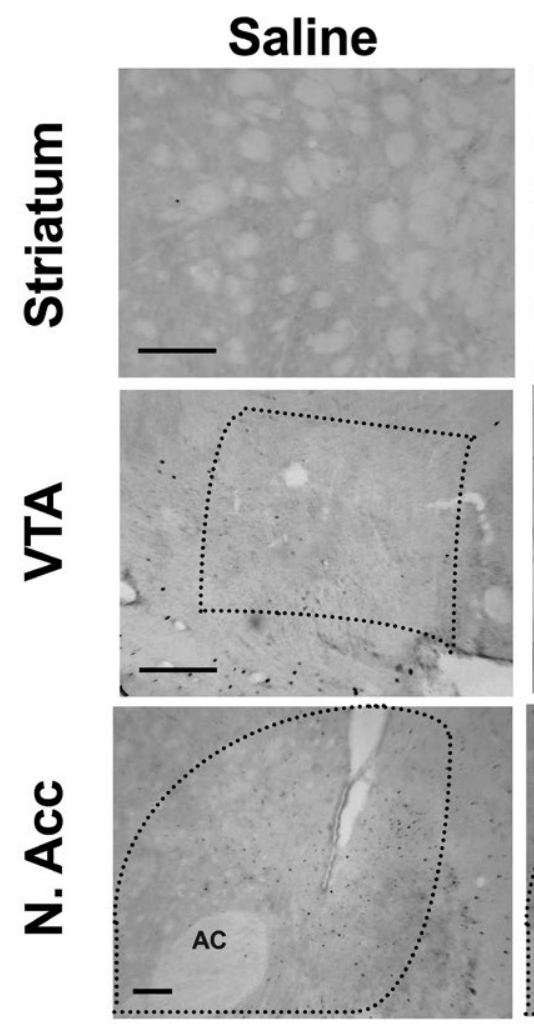

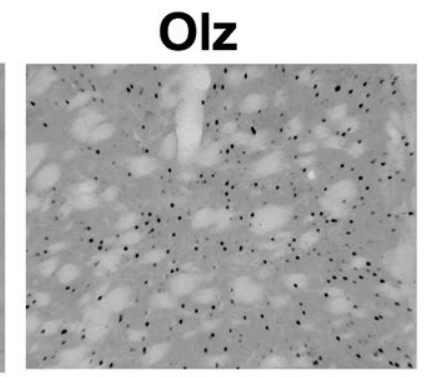

Olz + Mel
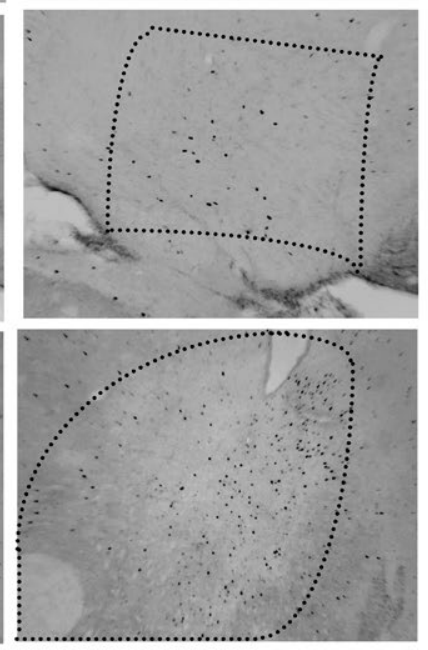
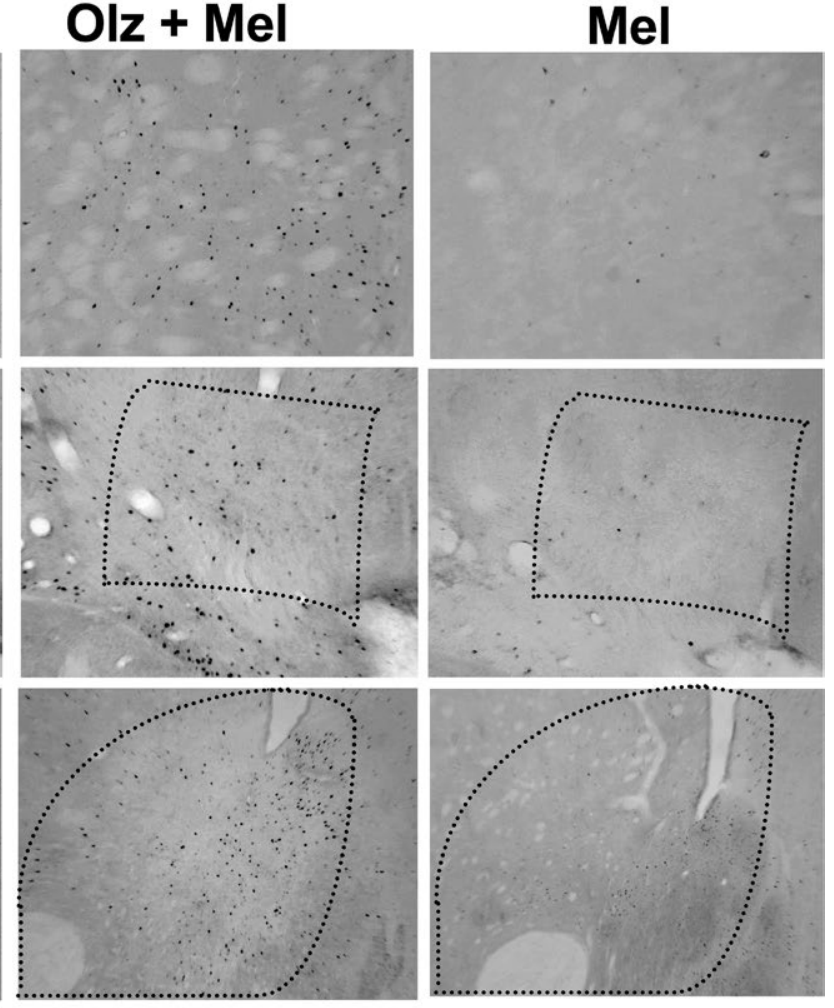

Striatum
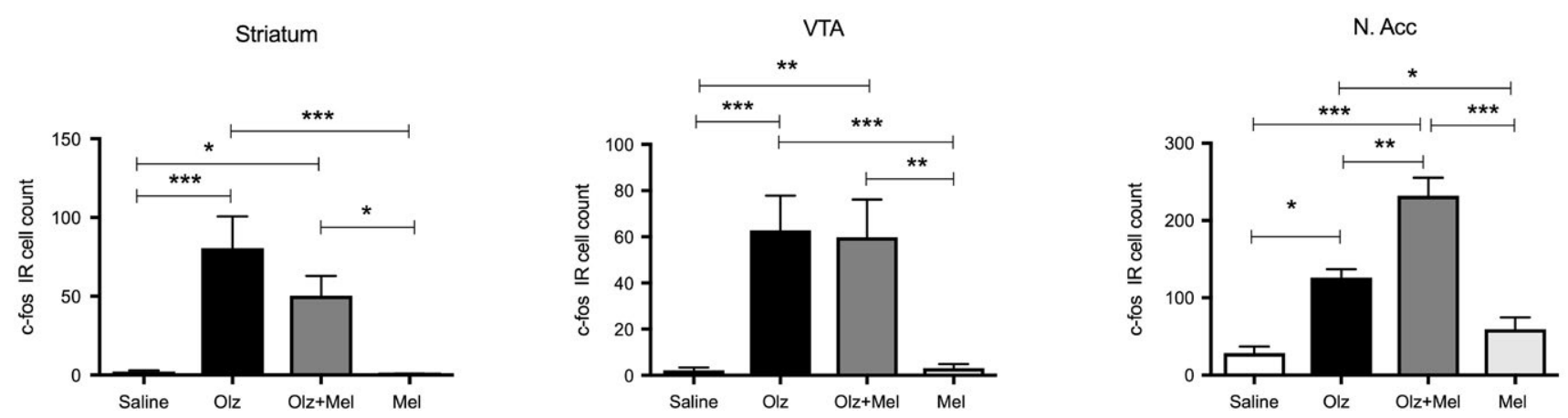

F I G URE 3 Olanzapine induced activation of brain regions associated with its therapeutic effect, not prevented by melatonin. Representative microphotographs of c-Fos immunoreactivity (IR) in the striatum (top), ventral tegmental area (VTA; middle row) and nucleus accumbens (N. Acc; bottom). The left column represents rats treated with saline, followed by a column of rats treated with olanzapine (Olz), olanzapine + melatonin $(\mathrm{Olz}+\mathrm{Mel})$ and melatonin only (Mel). $\mathrm{N}=4$ animals/group. Bars represent mean $\pm \mathrm{SEM}$ count for c-Fos IR nuclei in the striatum, VTA and N. acc. Anterior commissure (AC). One-way ANOVA analysis showed significant between-group differences for striatum $(F=13.79, d f=3, P<.0001)$, VTA $(F=15.78, d f=3, P<.0001)$, and N.Acc. $(F=25.19, d f=3, P<.0001$. $* P<.01 ; * * P<.001 ; * * *<.0001$; Bonferroni's post hoc test pair-wise comparisons. Scale bar is $100 \mu \mathrm{m}$

Parasympathetic hyperactivation may contribute to the development of metabolic syndrome, obesity ${ }^{39}$ or other SGA-induced adverse metabolic effects. Our anatomical analysis shows that olanzapine activates the parasympathetic branch of the ANS, with melatonin vastly mitigating this effect. To evaluate the functional significance of this finding, we measured the acute effects of olanzapine and melatonin on blood pressure and heart rate; two variables influenced by autonomic function and modified by olanzapine ${ }^{5,7}$. In agreement with the observed increase in activity of DMV neurons, olanzapine also induced a significant decrease in systolic, diastolic and mean arterial blood pressure (MAP), as well as in heart rate. The administration of melatonin ten minutes before the injection of olanzapine significantly attenuated its effect on systolic, diastolic, MAP and heart rate (Figure 4). Thus, olanzapine induces activation of the parasympathetic autonomic branch, decreasing blood pressure and heart rate; this is largely avoidable by giving melatonin. Next, and considering the high concentration of melatonin receptors in the $\mathrm{SCN}$, and not in PVN or $\mathrm{DMV}^{40}$, we hypothesized that the cardiovascular side effects of olanzapine, readily prevented by melatonin, were due to the effect of olanzapine on the SCN. To evaluate the involvement of the SCN in the 
Systolic blood pressure (SBP)

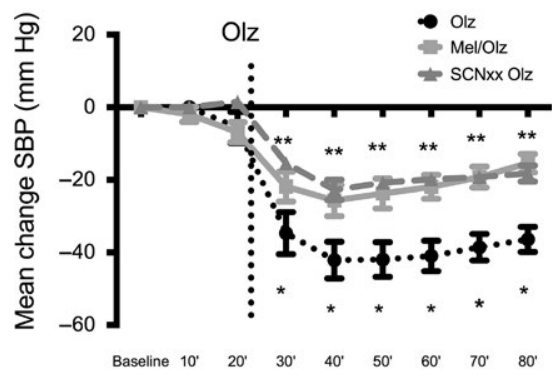

Diastolic blood pressure (DBP)

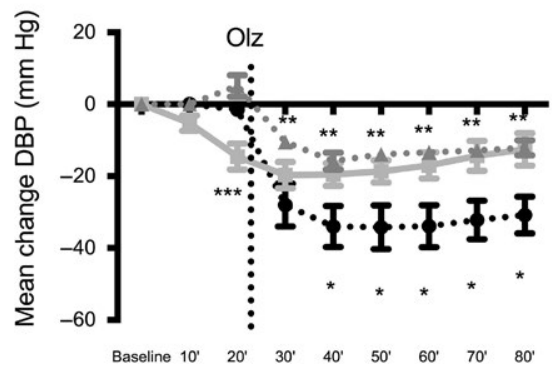

Mean arterial blood pressure (MAP)

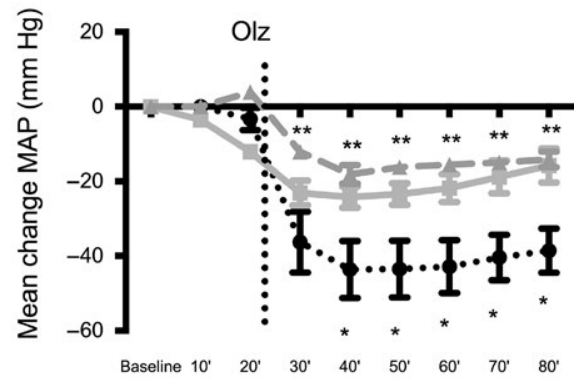

Heart rate (HR)

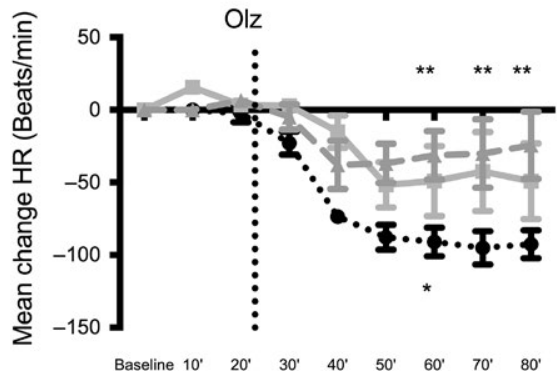

F I G U RE 4 Blood pressure and heart rate measurements. Olanzapine induced a decrease in systolic (SBP), diastolic (DBP) and mean arterial (MAP) blood pressure that was prevented by melatonin or a bilateral SCN lesion. Olanzapine also induced a decrease in heart rate (HR) that was counteracted by SCNxx and melatonin. Olanzapine (Olz); Melatonin/Olz (Mel/Olz); SCN lesioned animals injected with olanzapine (SCNxx). Hemodynamic parameters are reported in mean change from baseline. $\mathrm{N}=5$ animals/group. Repeated measures ANOVA showed significant treatment group $\mathrm{x}$ time interaction for Systolic $(F=3.216, d f=16, P<.0002)$, Diastolic $(F=9.561, d f=16, P<.0001)$, MAP analysis $(F=9.164, d f=16, P<.0001)$ and Heart rate $(F=2.089, d f=16, P=.014)$. Bonferroni's post hoc test for pair-wise comparison; $* P<.01 \mathrm{Olz}$ vs. Mel/Olz; $* * P<.01 \mathrm{Olz}$ vs. SCNxx Olz; $* * * P<.01 \mathrm{Mel} / \mathrm{Olz}$ vs. SCNxx Olz

hemodynamic effects of olanzapine, we performed bilateral SCN lesions ( $\mathrm{SCNxx}$ ) and after two weeks of recovery, we tested the effects of olanzapine on blood pressure and heart rate. Administration of olanzapine to SCNxx animals resulted in decreased effect on heart rate and blood pressure, similar to that observed in intact animals treated with olanzapine + melatonin (Figure 4 ). This observation confirms that olanzapine acts on the SCN to induce cardiovascular adverse effects and that these effects, associated with elevated comorbidity and mortality in SGA-treated patients, can be prevented by inhibiting the SCN activity with melatonin administration ${ }^{17-20}$.

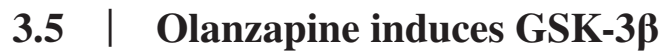 phosphorylation in the suprachiasmatic nucleus which is prevented by melatonin}

Consequently we investigated the intracellular mechanisms involved in the effects of olanzapine and melatonin in the SCN. Olanzapine has a wide spectrum of action and our results suggest that it could induce SCN neuronal activity via intracellular signaling cascades that converge with those of melatonin. Olanzapine induces phosphorylation of glycogen synthase kinase $3 \beta$ (GSK-3 $\beta$ ) and cAMP response element binding (CREB) protein via the Akt, Wnt, and PKC pathways, which are linked to its antipsychotic therapeutic and metabolic effects $^{41-44}$. Notably GSK-3 $\beta$ is involved in SCN function, neurogenesis, neurotransmission and metabolic processes ${ }^{45}$. Inhibition (phosphorylation) of GSK-3 $\beta$ triggers the activation (phosphorylation) of the cAMP response element binding protein (CREB), which in turn stimulates c-Fos transcription ${ }^{46,47}$. Interestingly, the phosphorylation of GSK-3 $\beta$ shows a circadian pattern, which is lowest at night ${ }^{45}$. In agreement with its action on the SCN whereby it reduces neuronal activity, melatonin decreases phosphorylation of GSK-3 $\beta$ via Akt1 ${ }^{48,49}$. Therefore, we evaluated the effects of olanzapine and melatonin on phosphorylated GSK-3 $\beta$ levels in the SCN. Indeed, olanzapine induced an increase in phosphorylated GSK-3 $\beta$ as compared to the control group and co-administration of melatonin reduced this effect. These findings provide a possible mechanism by which melatonin prevents the activation of SCN neurons by olanzapine. (Figure 5).

\section{4 | DISCUSSION}

Our animal experiments show that 1) olanzapine activates neurons in the SCN, PVN, and DMV; 2) melatonin prevents these effects; 3) pre-autonomic neurons in the PVN activated by olanzapine, project to the DMV and receive input from 
(A)

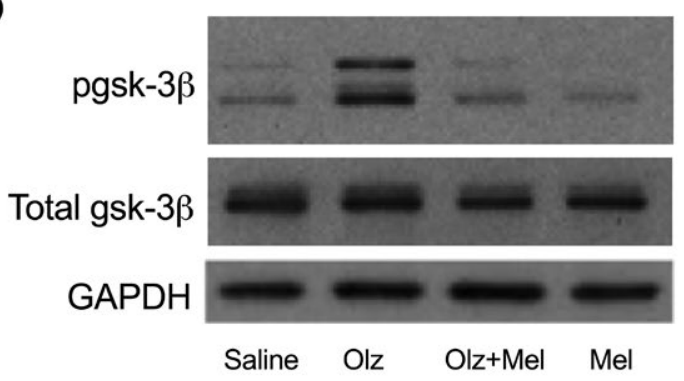

SCN (D)

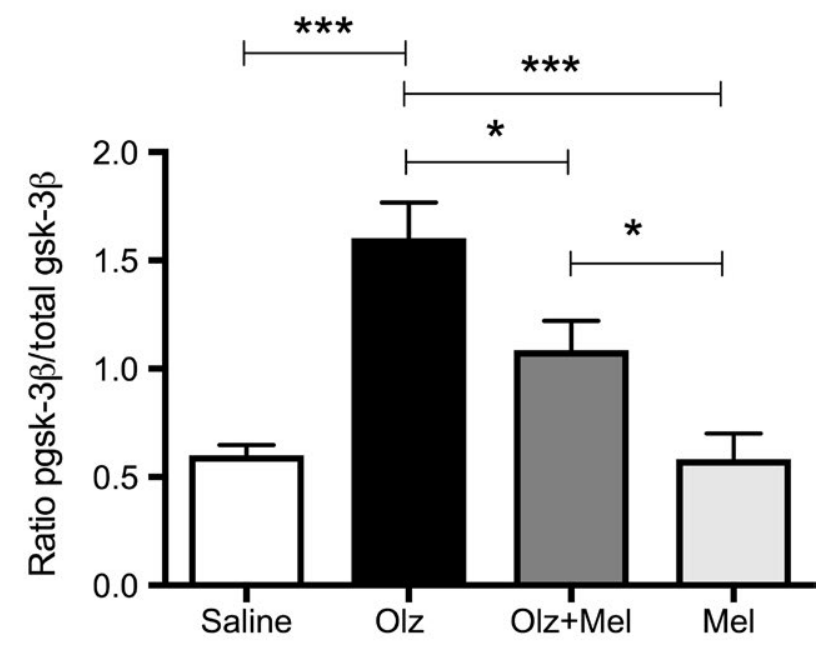

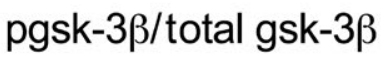

(B)

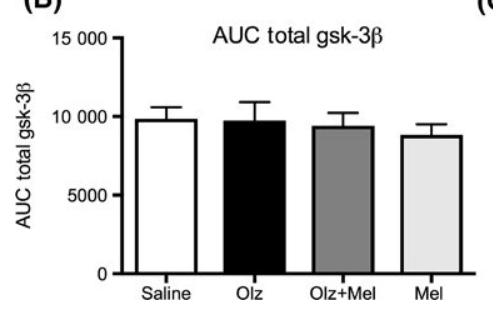

(C)

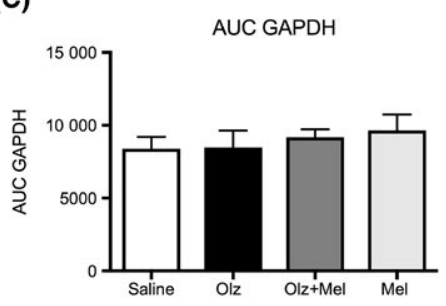

F I G U R E 5 Olanzapine induces the phosphorylation of GSK-3 $\beta$ in the SCN and this is prevented by melatonin. Representative Western blots of phosphorylated GSK-3 $\beta$ (pGSK-3 $\beta$ ) and total GSK-3 $\beta$ in homogenates of the SCN. Glyceraldheyde-3-phosphate dehydrogenase (GAPDH) was immunodetected as load control. (A) Neither total GSK-3 $\beta$ nor GAPDH levels were different between experimental groups (B and C). Densitometric analysis of the bands expressed as the ratio of the integrated optical density (IOD) of pGSK- $3 \beta$ and total pGSK-3 3 (pGSK- $3 \beta$ / Total pGSK- $3 \beta$ ratio) was performed for between group comparisons. Olanzapine increased pGSK-3 $\beta /$ total pGSK- $3 \beta$ ratio, which was reduced by melatonin (D). $\mathrm{N}=5$ animals/group. For: One-way ANOVA analysis showed a significant between- group difference in pGSK-3 $\beta /$ Total pGSK-3 $\beta$ ratio $(F=15.19, d f=3, P<.0001)$. $* P<.01 ; * * * P<.0001$; Bonferroni's post hoc test pairwise comparisons

the SCN; 4) olanzapine induces a decrease in blood pressure, prevented by melatonin; 5) the SCN is imperative to the cardiovascular side effects of olanzapine; 6) olanzapine induces inhibition of GSK-3 $\beta$ in the SCN that is reduced by melatonin, providing a possible intracellular mechanism for the cardiovascular effects of olanzapine and melatonin.

The biological clock selectively coordinates ANS balance to influence metabolism in different parts of the body, so alteration of this output may over time precipitate problems in metabolism such as the metabolic syndrome ${ }^{15}$. Acute parasympathetic activation induces adiposity and an increase in plasmatic adiponectin ${ }^{39}$. Such effects are also observed with short-term treatment with olanzapine ${ }^{50}$. With time, the increased parasympathetic activity induced by olanzapine favors the appearance of cardiometabolic adverse effects like obesity, as well as lipid, insulin and glucose disturbances ${ }^{1,4,51}$ similar to those observed in the metabolic syndrome; whereby in the long term, a compensatory increased sympathetic cardiovascular tone is reported ${ }^{1,15}$. These dynamic adjustments in autonomic activity may explain the increased sympathetic tone, increased blood pressure and decreased levels of adiponectin found after chronic use of SGAs such as olanzapine ${ }^{8,52}$. This might be a compensatory sympathetic effort of the ANS to counterbalance the chronic parasympathetic stimulus followed by the progressive appearance of metabolic disturbances induced by olanzapine. The immediate effect of melatonin, as we have demonstrated, prevents the increased parasympathetic output induced by olanzapine. Hereby melatonin may prevent short- and long-term ${ }^{17-20}$ hypothalamic and ANS disturbances induced by olanzapine, attenuating its adverse cardiometabolic effects. Antipsychotics act on diverse neurotransmitters in the brain $^{53}$, but are not known to act directly on melatonin receptors. Therefore, it is likely that melatonin prevents antipsychotic induced activation of the biological clock through shared intracellular mechanisms as the GSK-3B in the Akt-1 signaling pathway ${ }^{4-49}$. Based on this evidence, we propose a new model with a SCN-centered mechanism that could help explain SGAinduced cardiometabolic effects and the beneficial role of melatonin to prevent them. (Fig. S5) This study provides a new framework for the neuronal mechanisms involved in the cardiovascular and possibly other metabolic side effects of SGA and supports the rationale for the use of melatonin in a clinical setting to prevent them. Circulating melatonin may influence metabolic regulation through the SCN and could additionally have a direct peripheral action ${ }^{54,55}$. The SCN has already been shown to be linked with obesity and the metabolic syndrome, as well as hypertension. Our model could help to explain the role of the SCN and autonomic nervous system in early and chronic metabolic changes present in entities like obesity and the metabolic syndrome.

Collectively, present results and previous clinical data warrant additional basic and clinical investigation to demonstrate the SCN as a key region mediating the effects of antipsychotics on other acute and chronic metabolic changes, and show whether melatonin has opposing and potentially protective long-term effects. 


\section{ACKNOWLEDGEMENTS}

Authors thank Dr. Robert K. McNamara for his thoughtful comments. F.R.N. and R.M.B. conceived and designed the study protocol. F.R.N., R.M.B., F.N.B, C.E., M.C.B., M.V.T., G.B.K., and M.P. performed experimental techniques. All authors contributed to data analysis, discussion of results, writing, and revising the manuscript. This article is part of the Ph.D. thesis of F.R.N. (Ph.D. program in Biomedical Sciences at the Universidad Nacional Autónoma de México).

\section{CONFLICT OF INTEREST}

F.R.N. reports having received lecture fees from MOKSHA8 and research support from Productos Medix, S.A. de C.V. (México City, México) in a previous study. The authors of this work do not have commercial associations that might pose a conflict of interest in connection with this manuscript.

\section{REFERENCES}

1. Lieberman JA, Stroup TS, McEvoy JP, et al. Effectiveness of antipsychotic drugs in patients with chronic schizophrenia. N Engl J Med. 2005;353:1209-1223

2. Rummel-Kluge C, Komossa K, Schwarz S, et al. Head-to-head comparisons of metabolic side effects of second generation antipsychotics in the treatment of schizophrenia: A systematic review and meta-analysis. Schizophr Res. 2010;123:225-233.

3. Vidarsdottir S, de Leeuw van Weenen JE, Frolich M, Roelfsema F, Romijn JA, Pijl H. Effects of olanzapine and haloperidol on the metabolic status of healthy men. J Clin Endocrinol Metab. 2010;95:118-125.

4. Vancampfort D, Vansteelandt K, Correll CU, et al. Metabolic syndrome and metabolic abnormalities in bipolar disorder: A metaanalysis of prevalence rates and moderators. Am J Psychiatry. 2013; 170:265-274.

5. Choure BK, Gosavi D, Nanotkar S. Comparative cardiovascular safety of risperidone and olanzapine, based on electrocardiographic parameters and blood pressure: A prospective open label observational study. Indian J Pharmacol. 2014;46:493-497.

6. Markowitz JS, DeVane CL, Boulton DW, Liston HL, Risch SC. Hypotension and bradycardia in a healthy volunteer following a single $5 \mathrm{mg}$ dose of olanzapine. J Clin Pharmacol. 2002;42:104-106.

7. Leung JY, Pang CC, Procyshyn RM, Barr AM. Cardiovascular effects of acute treatment with the antipsychotic drug olanzapine in rats. Vascul Pharmacol. 2014;62:143-149.

8. McEvoy JP, Lieberman JA, Perkins DO, et al. Efficacy and tolerability of olanzapine, quetiapine, and risperidone in the treatment of early psychosis: A randomized, double-blind 52-week comparison. Am J Psychiatry. 2007;164:1050-1060

9. Westman J, Hallgren J, Wahlbeck K, Erlinge D, Alfredsson L, Osby U. Cardiovascular mortality in bipolar disorder: A populationbased cohort study in Sweden. BMJ Open. 2013;3:e002373.

10. Laursen TM, Wahlbeck K, Hallgren J, et al. Life expectancy and death by diseases of the circulatory system in patients with bipolar disorder or schizophrenia in the Nordic countries. PLOS ONE. 2013;8:e67133.
11. Hahn MK, Wolever TM, Arenovich T, et al. Acute effects of single-dose olanzapine on metabolic, endocrine, and inflammatory markers in healthy controls. J Clin Psychopharmacol. 2013;33:740-746.

12. Buijs RM, Escobar C, Swaab DF. The circadian system and the balance of the autonomic nervous system. Handb Clin Neurol. 2013;117:173-191

13. Takeda N, Maemura K. Circadian clock and the onset of cardiovascular events. Hypertens Res. 2016;39:383-390.

14. Buijs RM, Kalsbeek A. Hypothalamic integration of central and peripheral clocks. Nat Rev Neurosci. 2001;2:521-526.

15. Kreier F, Yilmaz A, Kalsbeek A, et al. Hypothesis: Shifting the equilibrium from activity to food leads to autonomic unbalance and the metabolic syndrome. Diabetes. 2003;52:2652-2656.

16. Liu C, Weaver DR, Jin X, et al. Molecular dissection of two distinct actions of melatonin on the suprachiasmatic circadian clock Neuron. 1997;19:91-102.

17. Raskind MA. Olanzapine-induced weight gain and increased visceral adiposity is blocked by melatonin replacement therapy in rats. Neuropsychopharmacology. 2007;32:284-288.

18. Romo-Nava F, Alvarez-Icaza Gonzalez D, Fresan-Orellana A, et al. Melatonin attenuates antipsychotic metabolic effects: An eight-week randomized, double-blind, parallel-group, placebocontrolled clinical trial. Bipolar Disord. 2014;16:410-421.

19. Modabbernia A, Heidari P, Soleimani R, et al. Melatonin for prevention of metabolic side-effects of olanzapine in patients with first-episode schizophrenia: Randomized double-blind placebocontrolled study. J Psychiatr Res. 2014;53:133-140.

20. Mostafavi A, Solhi M, Mohammadi MR, Hamedi M, Keshavarzi M, Akhondzadeh S. Melatonin decreases olanzapine induced metabolic side-effects in adolescents with bipolar disorder: A randomized double-blind placebo-controlled trial. Acta Med Iran. 2014;52:734-739.

21. Reppert SM, Weaver DR, Rivkees SA, Stopa EG. Putative melatonin receptors in a human biological clock. Science. 1988;242:78-81.

22. Dawe GS, Huff KD, Vandergriff JL, Sharp T, O'Neill MJ, Rasmussen K. Olanzapine activates the rat locus coeruleus: In vivo electrophysiology and c-Fos immunoreactivity. Biol Psychiatry. 2001;50:510-520.

23. Weston-Green K, Huang XF, Deng C. Alterations to melanocortinergic, GABAergic and cannabinoid neurotransmission associated with olanzapine-induced weight gain. PLoS ONE. 2012;7:e33548.

24. Kitagawa A, Ohta Y, Ohashi K. Melatonin improves metabolic syndrome induced by high fructose intake in rats. J Pineal Res. 2012;52:403-413.

25. Paxinos G, Watson C. The Rat Brain In Stereotaxic Coordinates. 6th ed. Amsterdam, Boston.: Academic Press/Elsevier; 2007.

26. Jespersen B, Knupp L, Northcott CA. Femoral arterial and venous catheterization for blood sampling, drug administration and conscious blood pressure and heart rate measurements. $J$ Vis Exp. 2012. doi: 10.3791/3496.

27. Buijs RM, Kalsbeek A, van der Woude TP, van Heerikhuize JJ, Shinn S. Suprachiasmatic nucleus lesion increases corticosterone secretion. Am J Physiol. 1993;264:R1186-R1192.

28. Lowry OH, Rosebrough NJ, Farr AL, Randall RJ. Protein measurement with the Folin phenol reagent. $J$ Biol Chem. 1951;193:265-275

29. Laemmli UK. Cleavage of structural proteins during the assembly of the head of bacteriophage T4. Nature. 1970;227:680-685. 
30. Towbin H, Staehelin T, Gordon J. Electrophoretic transfer of proteins from polyacrylamide gels to nitrocellulose sheets: Procedure and some applications. Proc Natl Acad Sci USA. 1979;76:4350-4354.

31. Alegria-Schaffer A. Western blotting using chemiluminescent substrates. Methods Enzymol. 2014;541:251-259.

32. Vidarsdottir S, Roelfsema F, Frolich M, Pijl H. Olanzapine shifts the temporal relationship between the daily acrophase of serum prolactin and cortisol concentrations rhythm in healthy men. Psychoneuroendocrinology. 2009;34:705-712.

33. Iwahana E, Akiyama M, Miyakawa K, et al. Effect of lithium on the circadian rhythms of locomotor activity and glycogen synthase kinase-3 protein expression in the mouse suprachiasmatic nuclei. Eur J Neurosci. 2004;19:2281-2287.

34. Buijs RM, la Fleur SE, Wortel J, et al. The suprachiasmatic nucleus balances sympathetic and parasympathetic output to peripheral organs through separate preautonomic neurons. J Comp Neurol. 2003;464:36-48.

35. Lacoste B, Angeloni D, Dominguez-Lopez S, et al. Anatomical and cellular localization of melatonin MT1 and MT2 receptors in the adult rat brain. J Pineal Res. 2015;58:397-417.

36. Robertson GS, Fibiger HC. Effects of olanzapine on regional C-Fos expression in rat forebrain. Neuropsychopharmacology. 1996; 14:105-110.

37. Sebens JB, Koch T, Ter Horst GJ, Korf J. Olanzapine-induced Fos expression in the rat forebrain; cross-tolerance with haloperidol and clozapine. Eur J Pharmacol. 1998;353:13-21.

38. Kreier F, Kap YS, Mettenleiter TC, et al. Tracing from fat tissue, liver, and pancreas: A neuroanatomical framework for the role of the brain in type 2 diabetes. Endocrinology. 2006;147:1140-1147.

39. Suzuki Y, Shimizu H, Ishizuka N, et al. Vagal hyperactivity due to ventromedial hypothalamic lesions increases adiponectin production and release. Diabetes. 2014;63:1637-1648.

40. Weaver DR, Stehle JH, Stopa EG, Reppert SM. Melatonin receptors in human hypothalamus and pituitary: Implications for circadian and reproductive responses to melatonin. J Clin Endocrinol Metab. 1993;76:295-301.

41. Pavan C, Vindigni V, Michelotto L, et al. Weight gain related to treatment with atypical antipsychotics is due to activation of PKC-beta. Pharmacogenomics J. 2010;10:408-417.

42. Lee JG, Cho HY, Park SW, Seo MK, Kim YH. Effects of olanzapine on brain-derived neurotrophic factor gene promoter activity in SH-SY5Y neuroblastoma cells. Prog Neuropsychopharmacol Biol Psychiatry. 2010;34:1001-1006.

43. Girgis RR, Javitch JA, Lieberman JA. Antipsychotic drug mechanisms: Links between therapeutic effects, metabolic side effects and the insulin signaling pathway. Mol Psychiatry. 2008;13:918-929.

44. Aubry JM, Schwald M, Ballmann E, Karege F. Early effects of mood stabilizers on the Akt/GSK-3beta signaling pathway and on cell survival and proliferation. Psychopharmacology. 2009;205:419-429.
45. Iitaka C, Miyazaki K, Akaike T, Ishida N. A role for glycogen synthase kinase-3beta in the mammalian circadian clock. J Biol Chem. 2005;280:29397-29402.

46. Grimes CA, Jope RS. CREB DNA binding activity is inhibited by glycogen synthase kinase- 3 beta and facilitated by lithium. $J$ Neurochem. 2001;78:1219-1232.

47. Ginty DD, Bonni A, Greenberg ME. Nerve growth factor activates a Ras-dependent protein kinase that stimulates c-fos transcription via phosphorylation of CREB. Cell. 1994;77: 713-725.

48. Ge D, Dauchy RT, Liu S, et al. Insulin and IGF1 enhance IL17-induced chemokine expression through a GSK3B-dependent mechanism: A new target for melatonin's anti-inflammatory action. J Pineal Res. 2013;55:377-387.

49. Mao L, Dauchy RT, Blask DE, et al. Circadian gating of epithelialto-mesenchymal transition in breast cancer cells via melatoninregulation of GSK3beta. Mol Endocrinol. 2012;26:1808-1820.

50. Togo T, Kojima K, Shoji M, et al. Serum adiponectin concentrations during treatment with olanzapine or risperidone: A pilot study. Int Clin Psychopharmacol. 2004;19:37-40.

51. Leucht S, Cipriani A, Spineli L, et al. Comparative efficacy and tolerability of 15 antipsychotic drugs in schizophrenia: A multiple-treatments meta-analysis. Lancet. 2013;382: 951-962.

52. Bartoli F, Lax A, Crocamo C, Clerici M, Carra G. Plasma adiponectin levels in schizophrenia and role of second-generation antipsychotics: A meta-analysis. Psychoneuroendocrinology. 2015;56:179-189.

53. Kuroki T, Nagao N, Nakahara T. Neuropharmacology of secondgeneration antipsychotic drugs: A validity of the serotonindopamine hypothesis. Prog Brain Res. 2008;172:199-212.

54. Cipolla-Neto J, Amaral FG, Afeche SC, Tan DX, Reiter RJ. Melatonin, energy metabolism, and obesity: A review. J Pineal Res. 2014;56:371-381.

55. Szewczyk-Golec K, Wozniak A, Reiter RJ. Inter-relationships of the chronobiotic, melatonin, with leptin and adiponectin: Implications for obesity. J Pineal Res. 2015;59:277-291.

\section{SUPPORTING INFORMATION}

Additional Supporting Information may be found online in the supporting information tab for this article.

How to cite this article: Romo-Nava F, Buijs FN, Valdés-Tovar M, et al. Olanzapine-induced early cardiovascular effects are mediated by the biological clock and prevented by melatonin. J. Pineal Res. 2017;62:e12402. https://doi.org/10.1111/jpi.12402 